\title{
Modélisation de l'interception des rayonnements solaires dans une culture en rangs. III. Application à une vigne traditionnelle
}

\author{
H Sinoquet ${ }^{1}$, C Valancogne ${ }^{2}$, A Lescure $^{1}$, R Bonhomme ${ }^{3}$ \\ 'INRA Antilles-Guyane, station agropédoclimatique, unité de bioclimatologie BP 1232, F97185 Pointe-à-Pitre Cedex; \\ ${ }^{2}$ INRA La Grande Ferrade, station de bioclimatologie, BP 81, F33883 Villenave-d'Ornon cedex; \\ ${ }^{3}$ INRA Paris-Massy-Grignon, station de bioclimatologie, F78850 Thiverval-Grignon France
}

(Reçu le 11 février 1991; accepté le 24 février 1992)

\begin{abstract}
Résumé - Un modèle d'interception du rayonnement solaire décrit précédemment est appliqué à un couvert spatialement discontinu: une vigne traditionnelle sur laquelle ont été mesurés les termes classiques du bilan radiatif et la distribution spatiale du rayonnement transmis au sol. La comparaison des mesures au modèle révèle un ajustement satisfaisant pour le rayonnement réfléchi (fig 4), mais assez médiocre pour le rayonnement transmis moyen (fig 5). Ceci est expliqué par l'utilisation de capteurs ponctuels qui, en raison de leur taille, mesurent plus souvent le rayonnement reçu au sol dans les taches de soleil ou les zones d'ombre qu'un rayonnement moyen. Ceci est vérifié en comparant les distributions mesurées et calculées du rayonnement transmis au sol (figs 7 et 8). Enfin, l'influence des parties ligneuses, non prise en compte dans le modèle, est clairement identifiée: elles réduisent sensiblement la transmission des rayonnements incidents (fig 9), et ceci d'autant plus que les capteurs sont proches du rang de végétation.
\end{abstract}

bilan radiatif / modélisation / couvert discontinu /Vitis vinifera = vigne

Summary - Modeling solar radiation interception in row plantation. III. Application to a traditional vineyard. A previously described model of solar radiation interception was applied to a spatially discontinuous canopy: that of a traditional vineyard in which the classical terms of the radiative balance and the spatial distribution of the radiation transmitted to the soil were measured. Comparison of measured and simulated data gave satisfactory agreement for reflected radiation (fig 4), but major discrepancies appeared for mean transmitted radiation (fig5). The use of small stationary sensors for measuring the transmitted radiation explains the latter observation, since most of the time they measured radiation received on the ground in the sunflecks or in the shaded area rather than mean radiation. This was verified by comparing the measured and simulated spatial distribution of transmitted radiation (figs 7,8 ). Finally, the influence of the woody parts which were not taken into consideration in the model was clearly identified: it significantly reduced the transmission of incident radiation (fig 9), and to a greater degres the closer the sensor was to the vegetation row.

radiative balance $/$ modelling $/$ discontinuous canopy $/$ Vitis vinifera $=$ vine 


\section{INTRODUCTION}

Introduite par Monsi et Saeki (1953), la modélisation des transferts radiatifs dans les couverts végétaux a d'abord été appliquée aux cultures homogènes, cultures ne présentant pas de variations de leurs caractéristiques dans le plan horizontal. Ce type de couvert a véritablement servi de base au développement de la théorie de l'interception du rayonnement au sein de la végétation. Amorcée par la mise en évidence de l'atténuation exponentielle du rayonnement en fonction de l'épaisseur de feuillage traversée (Monsi et Saeki, 1953), elle s'est poursuivie par la formalisation mathématique du coefficient d'extinction en fonction des caractéristiques du feuillage: d'abord, l'inclinaison des feuilles (Anderson, 1966; Chartier, 1966; Warren Wilson, 1967; entre autres), puis l'agencement spatial du feuillage (Acock et al, 1970; Nilson, 1971). Ce dernier paramètre décrit globalement la position des feuilles les unes par rapport aux autres, et donc leur tendance à s'ombrager mutuellement. L'ensemble des bases de cette théorie est présenté dans le travail de Nilson (1971).

La modélisation des échanges radiatifs dans les couverts hétérogènes, en particulier les cultures en rangs, a été abordée par 2 voies différentes:

- dans l'approche géométrique, les plantes ou ensembles de plantes (par exemple, les rangs) sont schématisés par des formes géométriques distribuées régulièrement dans l'espace. Sont alors étudiés l'interception du rayonnement par ces objets, et éventuellement les échanges radiatifs entre formes. Deux de ces modèles ont été appliqués au cas de la vigne dont les rangs sont alors assimilés à des parallélépipèdes longs (Smart, 1973; Riou et al, 1989). En plus de l'interception des rayonnements incidents, ces modèles considèrent les rayonnements réfléchis au niveau des parois des rangs. Le modèle de Riou et al (1989) prend aussi en compte la transmission des rayonnements à travers les rangs, en considérant que l'atténuation du rayonnement est proportionnelle à l'épaisseur de feuillage traversée;

- la seconde approche consiste à étendre aux cultures hétérogènes le formalisme classique des transferts radiatifs en couvert homogène. Là encore, 2 types d'approche peuvent être distingués:
- dans la première, le volume strictement occupé par le feuillage d'une plante ou d'un ensemble de plantes est assimilé à une forme géométrique à l'intérieur de laquelle les échanges radiatifs sont traités par la théorie classique. Les formes choisies pour représenter des rangs de végétation sont le plus souvent des cylindres horizontaux longs à section rectangulaire (Allen 1974; Mutsaers, 1980). Cette méthode se distingue de l'approche purement géométrique puisque les transferts sont traités au niveau des éléments de feuillage et non pas au niveau de l'enveloppe du volume de végétation;

- la seconde solution considère l'ensemble de l'espace compris entre le sol et le sommet de la végétation. Cet espace est virtuellement découpé en zones supposées homogènes, et les lois classiques de l'interception du rayonnement sont appliquées localement à chaque zone. Des modèles de ce type appliqués aux cultures en rangs ont été développés par Fukai et Loomis (1976), Cohen et Fuchs (1987) et Sinoquet (1989).

Ce dernier modèle a été testé de différentes façons: d'une part, il a été comparé au modèle de Bonhomme et Varlet-Grancher (1977) qui s'applique aux couverts homogènes (Sinoquet, 1989); d'autre part, il a été validé par des mesures de rayonnement transmis et réfléchi effectuées sur des cultures de maïs de structures variées (Sinoquet, 1988; Sinoquet et Bonhomme, 1989).: cultures en rangs d'orientation est-ouest et nordsud, alternance de rangs semés à 2 dates différentes de façon à accroître l'hétérogénéité du couvert.

L'application à une vigne traditionnelle, réalisée à partir de données collectées par Riou et al (1989), constitue un nouveau test du modèle à 2 niveaux: d'une part, la vigne étant un couvert franchement discontinu, ce travail doit permettre de juger s'il est possible d'étendre le champ d'application du modèle à des couverts présentant des hétérogénéités spatiales très marquées. D'autre part, les mesures de rayonnement de Riou et al (1989) contiennent des informations sur la distribution du rayonnement transmis au sol le long d'un transect perpendiculaire à la direction des rangs. C'est donc l'occasion d'évaluer l'aptitude du modèle à simuler non seulement les termes classiques du bilan radiatif (rayonnements moyens réfléchi et transmis) mais aussi la distribution spatiale du rayonnement 
transmis au sol. Ce test est plus sévère pour le modèle car il porte sur une description plus fine du microclimat lumineux de la culture. C'est peut-être aussi une des raisons pour lesquelles les modèles d'interception du rayonnement par les cultures en rangs n'ont jamais été, à notre connaissance, validés à ce degré de précision.

\section{MATÉRIEL ET MÉTHODES}

\section{Présentation du modèle}

\section{Interception des rayonnements par le feuillage}

Le modèle a été décrit de façon complète dans le premier article de cette série (Sinoquet, 1989). Comme indiqué plus haut, l'espace occupé par la végétation est découpé en couches horizontales et en tranches verticales parallèles à la direction des rangs. L'intersection d'une couche et d'une tranche définit un cylindre long en section rectangulaire, appelé "tube". Les variations spatiales de la structure géométrique du couvert sont prises en compte en affectant une densité de surface foliaire et une distribution d'inclinaisons à chaque tube. II s'agit donc d'une représentation bidimensionnelle du couvert, puisqu'il est supposé être homogène dans la direction parallèle aux rangs. Enfin, la succession des tranches verticales présente une évolution périodique, car la culture est supposée formée d'un ensemble de rangs identiques.

Les rayonnements direct, diffus et rediffusé par le sol et les plantes, sont décomposés en rayons élémentaires. La succession des tubes parcourus par un rayon et l'épaisseur de feuillage traversée dans chaque tube découlent de calculs géométriques simples. L'extinction du rayon au cours de son passage dans un tube est déterminée en appliquant localement les lois d'interception du rayonnement de Nilson (1971).

Cette procédure élémentaire permet de traiter les différents flux avec le même formalisme. L'interception du rayonnement direct est abordée en décomposant le rayonnement incident en une série de rayons parallèles. L'intégration des flux provenant de chaque rayon fournit le rayonnement direct intercepté. L'interception du rayonnement diffus est traitée de manière identique en discrétisant la voûte céleste en secteurs d'angle solide. Les rayons élémentaires, qui constituent le rayonnement rediffusé par un tube, sont issus de manière uniforme de chaque point du tube. Leur flux initial est déterminé par le champ directionnel de rayonnement rediffusé, fonction des propriétés du feuillage.
L'adimensionnement des flux permet de caractériser les échanges radiatifs par des facteurs de forme : ils représentent la part du rayonnement issu d'un émetteur, qui est interceptée par un récepteur. $\mathrm{Ne}$ dépendant que des propriétés géométriques du feuillage, ils sont l'expression de la structure du couvert, comme le sont les fréquences de trous dans le cas des couverts homogènes. Le bilan radiatif du couvert est établi en utilisant un formalisme inspiré de la méthode des radiosités (Ozisik, 1981) : les flux $I_{k}$ interceptés par chaque tube ou bande de sol $K$ sont les inconnues d'un système d'équations linéaires, dont les coefficients dépendent des facteurs de forme, des propriétés optiques des feuilles et du sol, et de l'intensité des flux incidents:

$$
I_{k}=S_{k}+D_{k}+\sum_{M=1}^{n k} f(M, K) \cdot \sigma_{M} \cdot I_{M}
$$

$S_{k}$ et $D_{k}$ correspondent à l'interception des flux incidents direct et diffus, alors que le dernier terme de l'équation représente l'interception des rayonnements rediffusés par les tubes et bandes de sol $M$ : en effet, par définition du coefficient de rediffusion $\sigma_{M}$, une partie $\sigma_{M} \cdot I_{M}$ du rayonnement $I_{M}$ est rediffusée dans l'espace par l'élément $M$; puis, par définition du facteur de forme $f(M, K)$ entre les échangeurs $M$ et $K$, une partie $f(M, K) \cdot \sigma_{M} \cdot I_{M}$ est interceptée par l'élément $K$. Finalement, ce formalisme permet d'obtenir les flux interceptés $I_{K}$, sans avoir à envisager, de façon artificielle, les rediffusions successives.

\section{Transmission du rayonnement dans les zones d'ombre et de soleil}

Le modèle d'origine a été légèrement modifié de façon à simuler aussi, pour chaque bande de sol, les rayonnements transmis dans les zones ombragées et ensoleillées. Les régions situées à l'ombre sont supposées ne recevoir que les rayonnements diffus et rediffusés moyens: le flux $l o_{k}$ transmis dans les taches d'ombre de la bande de sol $K$ s'écrit donc:

$$
l_{k}=D_{k}+\sum_{M=1}^{n k} f(M, K) \cdot \sigma_{M} \cdot I_{M}
$$

Par contre, les zones au soleil sont supposées recevoir les rayonnements diffus et rediffusés moyens plus l'intégralité du rayonnement incident direct: le flux $I s_{k}$ transmis par les parties ensoleillées de la bande de sol $K$ est:

$$
I_{k}=S_{o}+D_{k}+\sum_{M=1}^{n k} f(M, K) \cdot \sigma_{M} \cdot I_{M}
$$

où $S_{o}$ est le rayonnement direct au-dessus du couvert végétal. Enfin, la proportion de surface enso- 
leillée $S E_{k}$ au niveau de la bande de sol $K$ est égal à la transmittance du rayonnement direct (Chartier, 1986). On a donc:

$$
S E_{k}=S_{k} / S_{o}
$$

À partir des relations (1), (2), (3) et (4), on vérifie aisément que le rayonnement transmis moyen $I_{k}$ correspond bien à une pondération des flux $l_{k}$ et $l s_{k}$ par les surfaces ombragées et ensoleillées.

D'abord implanté sur un gros système (DPS8/62, Honeywell Bull), ce modèle complété fonctionne maintenant sur microordinateur compatible IBMPC.

\section{Interception des rayonnements par les parties ligneuses}

Contrairement aux éléments de feuillage, les parties ligneuses les plus grosses sont disposées de façon assez régulière, tout du moins dans le cas d'espèces cultivées. C'est pourquoi leur action sur les échanges radiatifs a été évaluée par une approche purement géométrique. Les pieds de vigne sont assimilés à des cylindres verticaux de hauteur $H$ et diamètre $d$ (fig 1 ). Une distance $D$ sépare 2 pieds successifs d'un même rang tandis que l'écartement des rangs est $L$. Le rayonnement issu d'une direction quelconque $\Omega$ - caractérisée par une hauteur $h$ et un azimut $A z$ par rapport à la direction des rangs - est intercepté par chaque pied. Ceux-ci provoquent un ombrage sur le sol jusqu'à une distance $X$ sur l'axe perpendiculaire aux rangs (fig 1a):

$$
X=H \cdot \sin (A z) / \operatorname{tg}(h)
$$

D'autre part, l'intensité de l'ombrage dépend évidemment de la porosité $p$ de la ligne de pieds qui pourrait être définie par le rapport $(D-d) / D$ :

\section{Ombrage créé par une ligne de pieds}

Dans la zone affectée par l'ombrage, le pourcentage de surface ombragée par les pieds d'un rang est (fig 1b) :

$$
S O_{p}=(d / D) / \sin (A z)=(1-p) / \sin (A z)
$$

Lorsque la relation (6) donne une valeur de $S O_{p}>1$, cela signifie que les ombres portées par plusieurs pieds successifs d'une même ligne se chevauchent, aucun rayon ne filtrant à travers la rangée de pieds. Comme les tiges sont opaques, la relation (6) peut être exprimée comme suit:

$$
S O_{p}=\operatorname{Min}[1,(1-p) / \sin (A z)]
$$

La surface de sol non concernée par l'ombrage des pieds est évidemment: $S E_{p}=1-S O_{p}$.

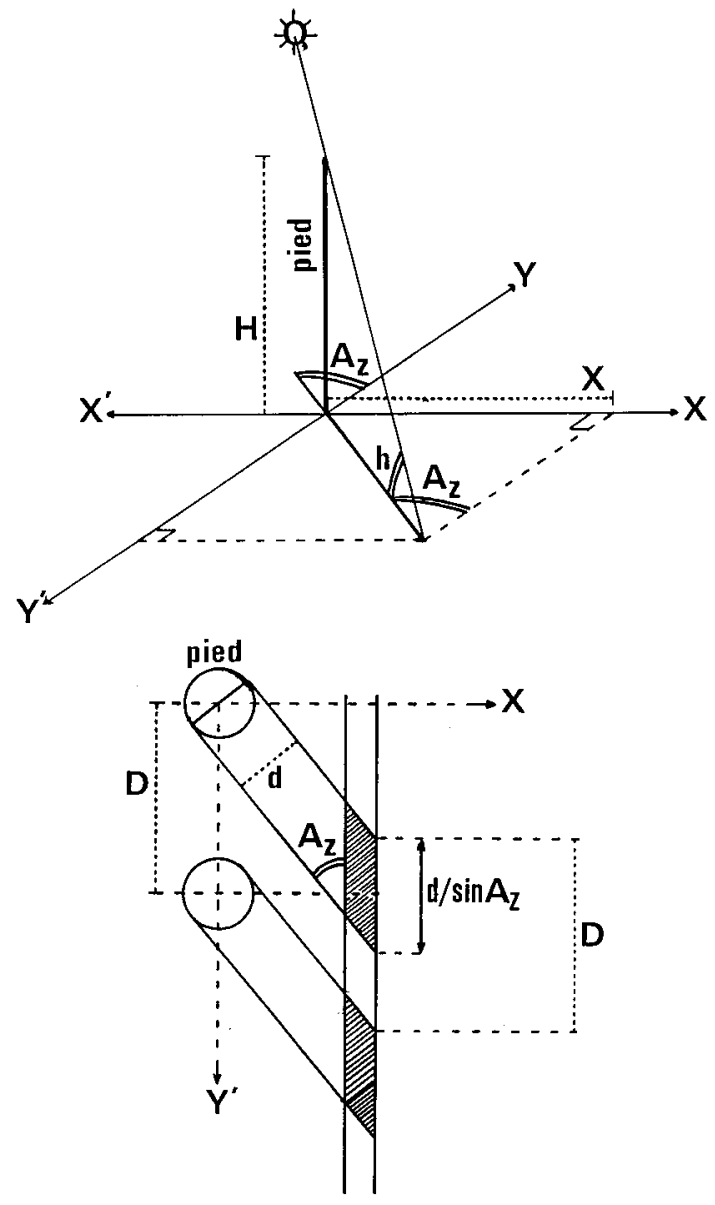

Fig 1. Représentation schématique de l'interception du rayonnement par les pieds. L'axe $X$ est perpendiculaire aux rangs, l'axe $Y$ est parallèle aux rangs. a. Ombre portée par un pied en fonction de sa hauteur $H$ et de la position du soleil (hauteur $h$, azimut $A z$ par rapport à l'axe $Y$ ). b. Surface de sol ombragée en fonction de la position du soleil et de la porosité des lignes de pieds (rapport $(D-d) / D)$

\section{Recouvrement des ombrages de plusieurs lignes de pieds}

Lorsque la zone subissant de l'ombrage est supérieure à la distance entre 2 rangs $(X>L)$, certaines bandes de sol sont ombragées par plusieurs lignes de pieds. On suppose alors qu'il y a indépendance entre la position des pieds d'une ligne à l'autre; ceci revient à effectuer le produit des termes $S E p$ de chaque rang provoquant de l'ombre.

De façon similaire, les ombres portées par le feuillage (relation 4) et les pieds sont supposées être indépendantes. Dans le cas du rayonnement direct, seule la direction du soleil est prise en compte et l'action principale des pieds consiste en une réduction de la quantité des taches de soleil. Dans le cas du rayonnement diffus, la voûte céleste est de nouveau découpée en secteurs d'angle solide et l'action des pieds est calculée en sommant la contribution de chaque secteur. Enfin, 
comme les parties ligneuses sont opaques et sombres, les rayonnements qu'elles rediffusent sont négligés.

Ces calculs ne sont pas directement intégrés dans le modèle de base. Ils sont seulement utilisés pour donner une première approximation de l'influence des parties ligneuses sur la distribution du rayonnement au sol.

\section{Dispositif expérimental}

La culture, implantée sur le domaine de Latresne, est une vigne traditionnelle de la région de Bordeaux. Le feuillage, taillé sévèrement, est contenu dans une enveloppe de forme parallélépipédique. Les caractéristiques de la culture ont été décrites par Riou et al (1989): la vigne est orientée nordsud; l'écartement des rangs est de $1,8 \mathrm{~m}$, la largeur des rangs est $0,3 \mathrm{~m}$; la hauteur de la culture est $1,5 \mathrm{~m}$ mais le feuillage n'est présent qu'entre les niveaux $0,6 \mathrm{~m}$ et $1,5 \mathrm{~m}$ ( $\mathrm{fig} 2$ ).

Le feuillage est supporté par des parties ligneuses: le pied de vigne, ou cep, est une branche plus ou moins verticale entre le sol et une hauteur de $50 \mathrm{~cm}$. II y a un cep/m linéaire de rang et leur diamètre a été estimé à $6 \mathrm{~cm}$. À partir de $50 \mathrm{~cm}$ au-dessus du sol, le cep, qui peut prendre une direction horizontale, supporte une dizaine de branches plus fines appelées sarments. Ceux-ci montent jusqu'au sommet de la végétation. Leur diamètre à la base est de l'ordre de $2 \mathrm{~cm}$.

Riou et al (1989) ont mesuré le bilan radiatif de cette vigne du 25 au 30 septembre 1986 de la façon suivante: les rayonnements incidents global et diffus et le rayonnement réfléchi ont été mesurés avec des pyranomètres (Kipp et Zonen) fixés sur un mât à $3,5 \mathrm{~m}$ du sol. Quatre autres thermopiles placées au sol sur un transect perpendiculaire à la direction des rangs mesuraient le rayonnement global transmis; leur position exacte est indiquée sur la figure 2. Une moyenne des signaux délivrés par les capteurs était effectuée chaque quart d'heure.

\section{Paramétrisation du système}

\section{Structure géométrique du couvert végétal}

Les paramètres d'entrée décrivant la structure du couvert ont été déterminés à partir des informations fournies par Riou et al (1989) : la surface foliaire $\left(1,8 \mathrm{~m}^{2} / \mathrm{m}\right.$ linéaire de rang) n'est pas répartie uniformément dans le volume de végétation puisque les feuilles sont surtout présentes à proximité des parois du rang. D'autre part, l'inclinaison des feuilles proches des parois horizontales montre une tendance plutôt horizontale tandis que les parois verticales contiennent des feuilles plutôt verticales.

Pour rendre compte de cette structure, le volume total (rang + interrang, soit 1,5 $\mathrm{m} \times 1,8 \mathrm{~m} \times 1 \mathrm{~m}$ linéaire de rang) a été divisé en 270 cellules de taille $0,1 \mathrm{~m} \times 0,1 \mathrm{~m} \times 1 \mathrm{~m}$. L'affectation de la surface foliaire dans les 27 cellules correspondant au volume de végétation est décrite en figure 3 : seules les cellules de bordure contiennent de la surface foliaire. Elles ont été regroupées en 4 ensembles : les 2 parois verticales et les 2 parois horizontales. Chaque groupe reçoit une même quantité de surface foliaire, soit $0,45 \mathrm{~m}^{2}$, elle-même répartie uniformément entre les cellules du groupe. On aboutit ainsi à une densité de surface foliaire de $15 \mathrm{~m}^{2} \cdot \mathrm{m}^{-3}$ pour les parois horizontales et de $6,4 \mathrm{~m}^{2} \cdot \mathrm{m}^{-3}$ pour les parois verticales. Enfin, la distribution des inclinaisons a été supposée planophile (respectivement érectophile) dans les parois horizontales (respectivement verticales).

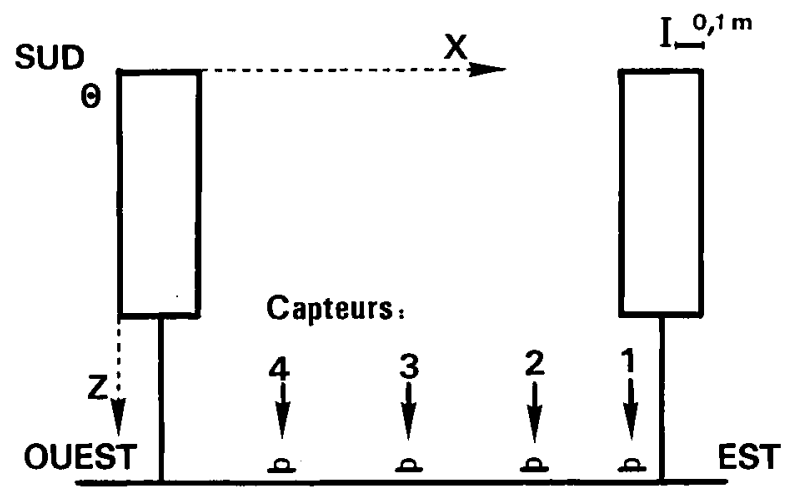

Fig 2. Représentation schématique de la culture et position des capteurs de rayonnement transmis au sol.

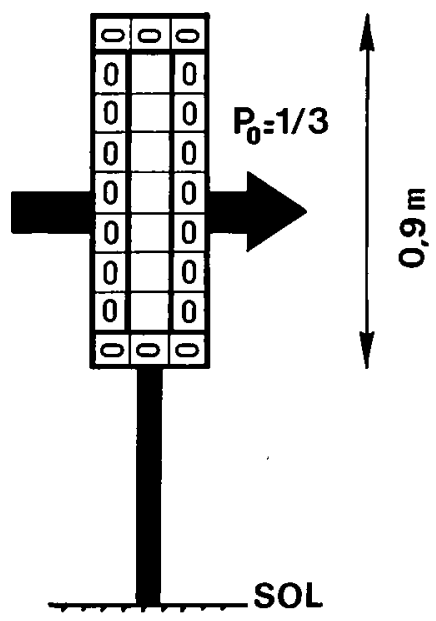

Fig 3. Illustration de la distribution de la surface foliaire dans le volume de végétation: les 27 cellules sont réparties en 5 groupes : la zone centrale est vide, les 2 parois horizontales comportent du feuillage planophile alors que celui des 2 parois verticales est érectophile. 
Cette paramétrisation est en accord avec la description de Riou et al (1989) qui indiquent que les parois horizontales sont très denses. D'autre part, elle conduit à une porosité du rang très proche de la valeur estimée par photographies numérisées. En effet, la relation qui relie fréquence de trous et structure du couvert peut s'écrire (Nilson, 1971):

$$
P O=\exp [-K \cdot S \cdot I F]
$$

où $K$ est le coefficient de projection de l'unité de surface foliaire, $S$ le paramètre d'agencement du feuillage et IF I'indice foliaire. Ici, l'agencement est supposé être aléatoire $(S=1)$. Appliquée à une direction horizontale perpendiculaire aux rangs, la relation (8) conduit à une fréquence de trous de $29 \%$, alors que la valeur mesurée est de $1 / 3$ (Riou et al, 1989). Cette convergence permet de supposer que les trouées dans la végétation sont bien prises en compte même si elles sont dues à des zones assez grandes où le feuillage est manquant.

\section{Propriétés optiques des feuilles et du sol}

Les propriétés optiques ont été mesurées avec un spectrophotomètre (LI-1800, LI-COR Inc) sur des feuilles de la même espèce et le même sol. Le spectre solaire a été divisé en 2 domaines de longueur d'onde pour lesquels les propriétés radiatives des feuilles sont contrastées : bande visible $(400-200 \mathrm{~nm})$ et bande proche infrarouge (700$1200 \mathrm{~nm})$, en supposant que chacune comprend la moitié de l'énergie incidente (Varlet-Grancher, 1975). Les valeurs moyennes des propriétés optiques pour ces domaines (tableau I) ont été obtenues en convoluant les valeurs spectrales avec la composition spectrale du rayonnement incident.

\section{RÉSULTATS ET DISCUSSION}

\section{Comparaison des rayonnements réfléchis}

La figure 4 présente la comparaison entre rayonnements réfléchis mesurés et simulés par le modèle. Les valeurs calculées sont assez proches de la première bissectrice, mais le plus souvent, elles sont placées au-dessus. La surestimation du modèle est plus nette pour les fortes valeurs de rayonnement réfléchi.

L'examen des résidus (ie, écarts entre valeurs simulées et valeurs mesurées) indique un écart maximal de $11 \mathrm{~W} \cdot \mathrm{m}^{-2}$. La moyenne des résidus, égale à $+3,5 \mathrm{~W} \cdot \mathrm{m}^{-2}$, confirme la légère surestimation systématique, tandis que leur écart type, égal à $3 \mathrm{~W} \cdot \mathrm{m}^{-2}$, confirme la faible dispersion des points. Malgré leur faible
Tableau I. Propriétés optiques des feuilles et du sol pour les domaines spectraux visible et proche infrarouge.

\begin{tabular}{|c|c|c|}
\hline & Visible & $\begin{array}{l}\text { Proche } \\
\text { infrarouge }\end{array}$ \\
\hline Feuille & & \\
\hline Réflectance & 0,075 & 0,40 \\
\hline Transmittance & 0,045 & 0,44 \\
\hline $\begin{array}{l}\text { Coefficient moyen } \\
\text { de rediffusion }\end{array}$ & 0,06 & 0,42 \\
\hline $\begin{array}{l}\text { Sol } \\
\text { Réflectance }\end{array}$ & 0,12 & 0,24 \\
\hline
\end{tabular}

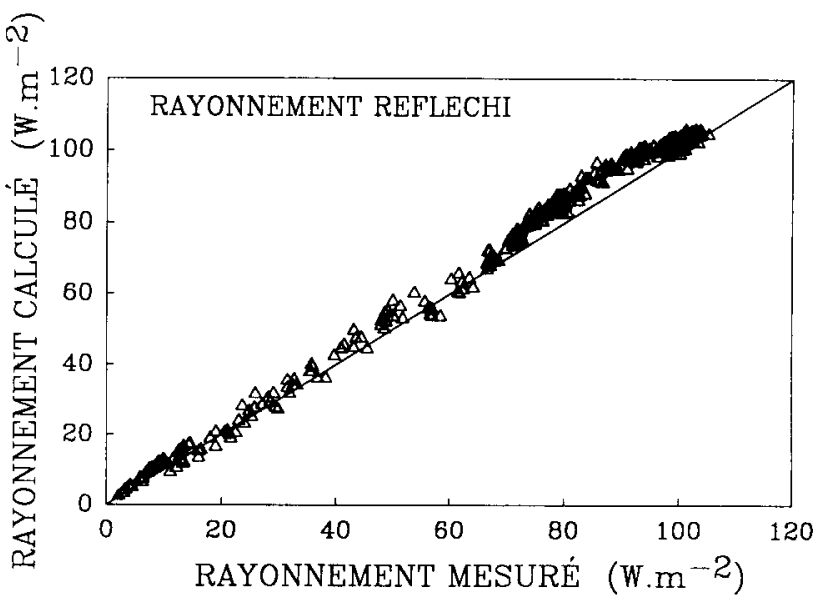

Fig 4. Comparaison des rayonnements réfléchis mesurés et calculés par le modèle.

valeur, les résidus sont assez dépendants de la hauteur du soleil et du rapport diffus/global (rapport des rayonnements incidents diffus et global) puisque les coefficients de corrélation, calculés sur l'ensemble des 250 points de mesure, sont respectivement 0,43 et $-0,34$. De plus, en ne considérant que les mesures effectuées par ciel clair (215 points), la corrélation entre résidus et hauteur du soleil passe à 0,48 .

Des relations identiques ont déjà été signalées (Sinoquet et Bonhomme, 1989) lors de l'application du modèle à des cultures de maïs. Elles avaient été attribuées à une mauvaise estimation de la pénétration directionnelle des rayonnements: dans la relation classique reliant la fréquence de trous à la structure du couvert, l'agencement du feuillage est souvent considéré comme indépendant de la direction de pénétration du rayonnement alors que des variations sont tout à fait possibles. Dans le cas du maïs, Prévot (1985) a effectué un ajustement de la 
relation avec un paramètre $S$ constant sur des mesures directionnelles de fréquences de trous réalisées grâce à des photographies hémisphériques. II aboutit à une surestimation de la fréquence de trous pour les faibles hauteurs du soleil et une sous-estimation pour les hauteurs proches du zénith. De telles observations, confirmées par ailleurs (Andrieu, 1989; com pers) signifient que l'agencement du feuillage varie avec la direction de pénétration du rayonnement: dans le cas du maïs, l'agencement est plus agrégatif pour les directions verticales que pour les incidences rasantes.

Dans ces conditions, la relation entre résidus et hauteur du soleil peut être expliquée par l'influence prépondérante de l'interception du rayonnement direct qui se révèle particulièrement les jours bien ensoleillés.

\section{Comparaison des rayonnements transmis}

\section{Comparaison des rayonnements transmis moyens}

La comparaison des moyennes spatiales des rayonnements transmis au sol est présentée en figure 5 . Elle révèle une nette surestimation des calculs qui, de plus, est associée à une dispersion assez importante: les écarts varient de $-40 \mathrm{~W} \cdot \mathrm{m}^{-2}$ à $+160 \mathrm{~W} \cdot \mathrm{m}^{-2}$; la moyenne des résidus est de l'ordre de $+50 \mathrm{~W} \cdot \mathrm{m}^{-2}$ alors que leur écart type est égal à $35 \mathrm{~W} \cdot \mathrm{m}^{-2}$. Pour expliquer ces résultats assez médiocres, 3 hypothèses de désaccord possible ont été testées successivement:

$H_{1}$ Les 4 capteurs placés au sol effectuentils une réelle moyenne spatiale du rayonnement transmis?

$\mathrm{H}_{2}$ Les capteurs de rayonnement transmis, ponctuels et fixes, mesurent-ils réellement le rayonnement transmis moyen de la bande de sol dans laquelle ils sont situés?

$\mathrm{H}_{3}$ Quelle est l'influence des parties ligneuses sur les rayonnements transmis au sol?

Le test de l'hypothèse $H_{1}$ a consisté à vérifier, par simulation, que le rayonnement moyen transmis au sol était estimé de façon correcte par la moyenne des rayonnements reçus par les bandes de sol portant les thermopiles. En effet, d'une part, le modèle permet de simuler le rayonnement transmis au niveau de chaque bande de sol; d'autre part, le rayonnement transmis moyen a été estimé par la moyenne des mesures fournies par les 4 capteurs situés sur le sol. La figure 6 montre un très bon accord entre les valeurs simulées du rayonnement transmis moyenné sur l'ensemble des bandes de sol et la moyenne des valeurs simulées pour les 4 bandes de sol supportant les capteurs. L'estimation ne comporte aucun biais, comme en témoigne la droite de régression:

$$
T_{\text {moy }}=0,999 \quad T_{T H P} \quad r^{2}=0,991 \quad N=250
$$

L'erreur d'estimation du rayonnement transmis moyen ( $\left.T_{\text {moy }}\right)$ à partir de la moyenne des flux reçus par les bandes de sols contenant les capteurs $\left(T_{T H P}\right)$ est de l'ordre de $15 \mathrm{~W} \cdot \mathrm{m}^{-2}$. Les écarts sont très petits pour les faibles valeurs de rayonnement, ils ont tendance à augmenter pour les valeurs les plus fortes pour

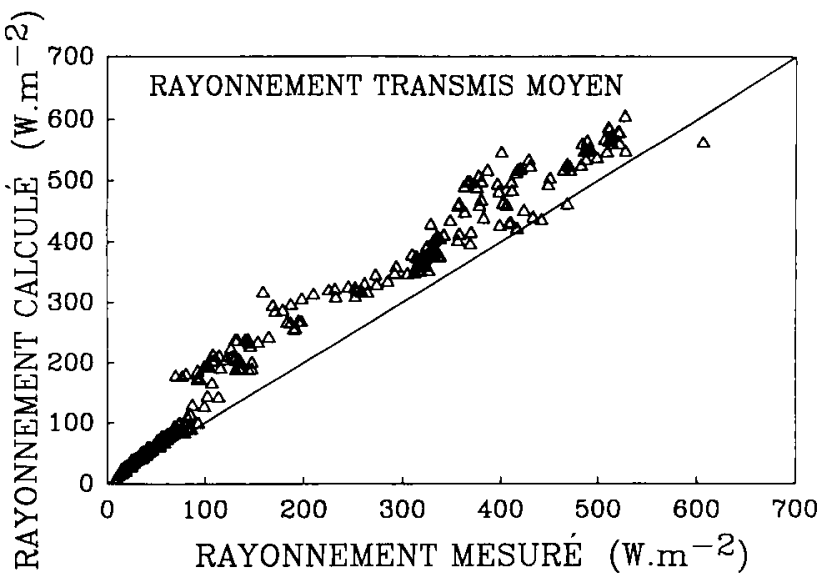

Fig 5. Comparaison des rayonnements moyens transmis au sol mesurés et calculés par le modèle.

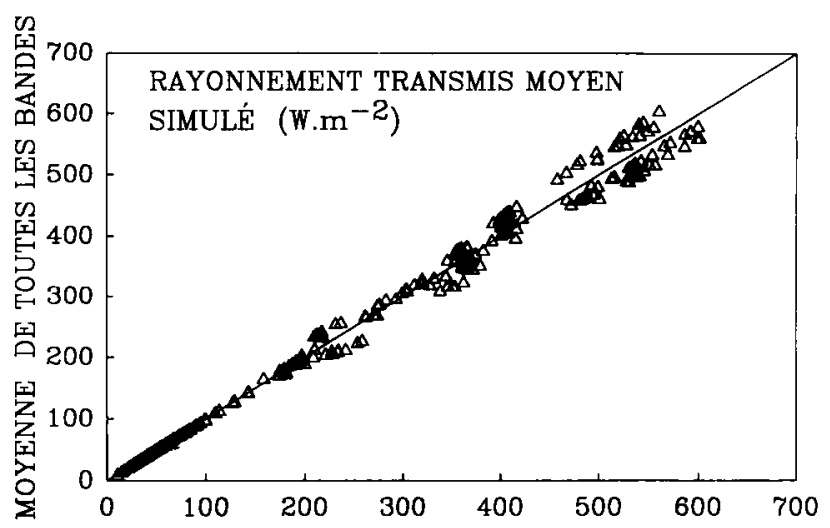

MOYENNE DES BANDES OCCUPÉES PAR LES CAPTEURS

Fig 6. Comparaison des rayonnements moyens transmis au sol simulés par le modèle: en abscisse, moyenne des valeurs simulées sur les bandes de sol occupées par les capteurs; en ordonnée, moyenne des valeurs simulées sur toutes les bandes de sol. 
atteindre au maximum $45 \mathrm{~W} \cdot \mathrm{m}^{-2}$. Cependant, ils sont insuffisants pour expliquer l'adéquation médiocre entre les rayonnements transmis mesurés et calculés de la figure 5 .

\section{Comparaison des rayonnements transmis au niveau de chaque capteur}

Cette comparaison a deux objectifs: d'une part, identifier l'aptitude du modèle à décrire la distribution spatiale du rayonnement transmis au sol; d'autre part, répondre aux questions $\mathrm{H}_{2}$ et $\mathrm{H}_{3}$ concernant les écarts observés au niveau des rayonnements transmis moyens.

\section{Zones d'ombre et taches de soleil}

Compte tenu du caractère ponctuel des thermopiles, de leur position fixe et du rapport entre leur surface réceptrice et celle des feuilles de vigne, l'hypothèse $\mathrm{H}_{2}$ a consisté à envisager que les capteurs placés au sol puissent être situés, soit dans des taches de soleil, soit dans des zones d'ombre.
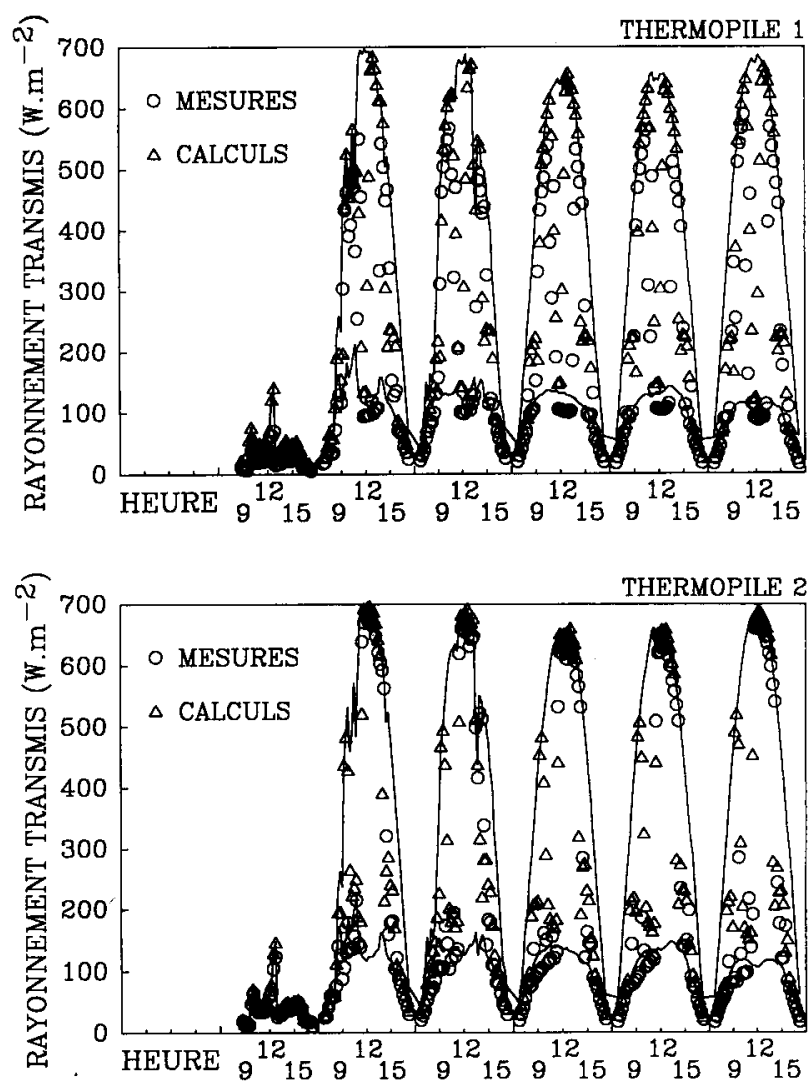

La figure 7 présente l'évolution en fonction du temps des rayonnements transmis mesurés au niveau de chaque thermopile ainsi que des valeurs simulées de 3 types: rayonnement reçu dans les taches d'ombre, rayonnement reçu dans les taches de soleil, rayonnement moyen reçu par la bande de sol portant le capteur. Malgré des variations selon les capteurs, on observe généralement une majorité de points de mesure proches des courbes simulées de rayonnement transmis en zone ombragée ou en zone au soleil. De façon corollaire, les points de mesure éloignés de ces courbes extrêmes sont peu nombreux, bien que cette tendance soit moins nette dans le cas de la thermopile 1. Par conséquent, il apparaît effectivement qu'une grande partie des points de mesure ont eu lieu lorsque les capteurs étaient totalement au soleil ou complètement ombragés.

Pour confirmer cette hypothèse, les rayonnements mesurés et calculés ont été comparés en appliquant un critère basé sur la proportion de surface ensoleillée $S E_{k}$ de la bande de sol $K$ : si $S E_{k}$ (relation 4) est inférieur à $50 \%$, le capteur est supposé être à l'ombre; dans le cas contraire, il est supposé
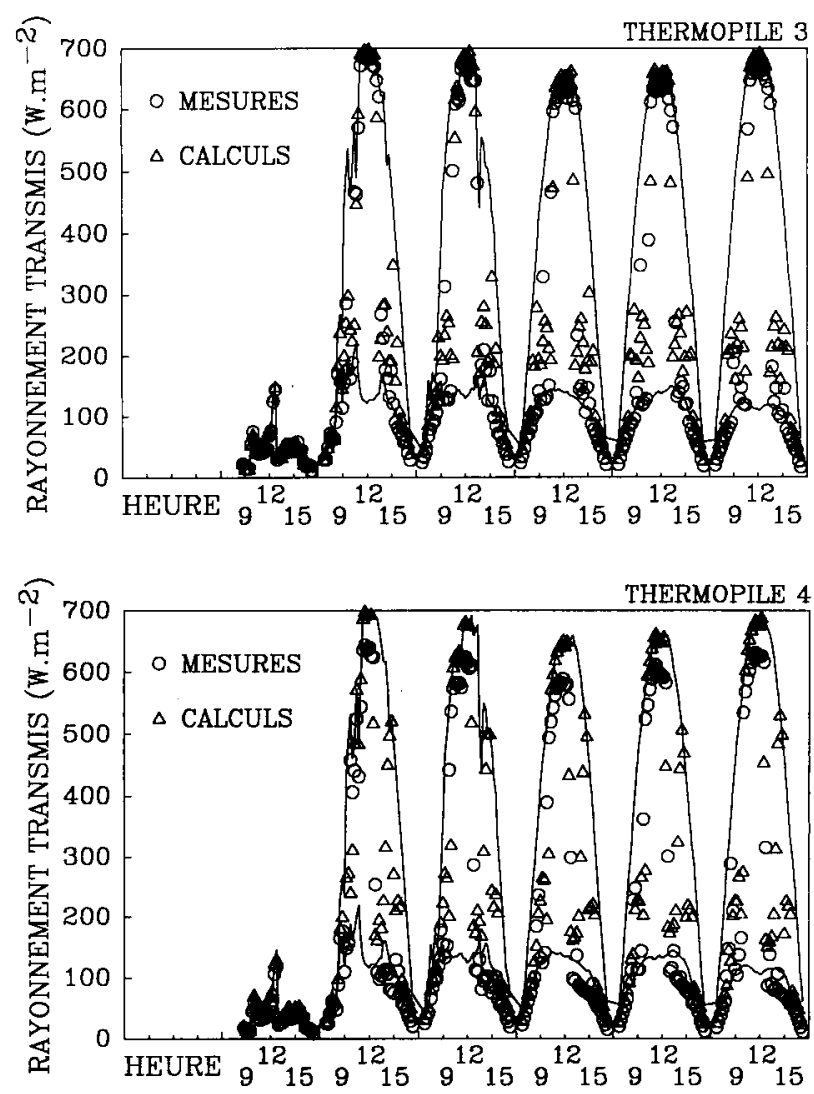

Fig 7. Évolution des rayonnements transmis mesurés et simulés au niveau de chaque capteur: la courbe enveloppe supérieure (resp inférieure) correspond aux valeurs simulées de rayonnement transmis dans les taches de soleil (resp dans les zones ombragées). a) Thermopile 1, b) Thermopile 2, c) Thermopile 3, d) Thermopile 4. 
situé dans une tache de soleil. Pour chacun des capteurs, les nuages de points ont vaguement l'aspect d'un " $Z$ " (fig 8): la majorité des points est située près de la première bissectrice et obéit donc à l'hypothèse de départ. La barre inférieure du " $Z$ " peut être attribuée aux points de mesure situés dans les taches de soleil ou partiellement ensoleillés alors que plus de $50 \%$ de la surface de sol est ombragée. Réciproquement, la barre supérieure du « $Z$ » pourrait correspondre aux points de mesure où le capteur est plus ou moins ombragé alors que plus de $50 \%$ de la surface du sol est au soleil.

La proportion de points de mesures qui satisfait l'hypothèse de travail (ie, les points proches de la première bissectrice) varie, en fonction du capteur, d'environ 75 à $90 \%$ (tableau II). II est cohérent de trouver des points ne satisfaisant pas cette condition: en effet, les capteurs peuvent se trouver au soleil, alors que la majorité de la surface du sol est ombragée, et vice versa.
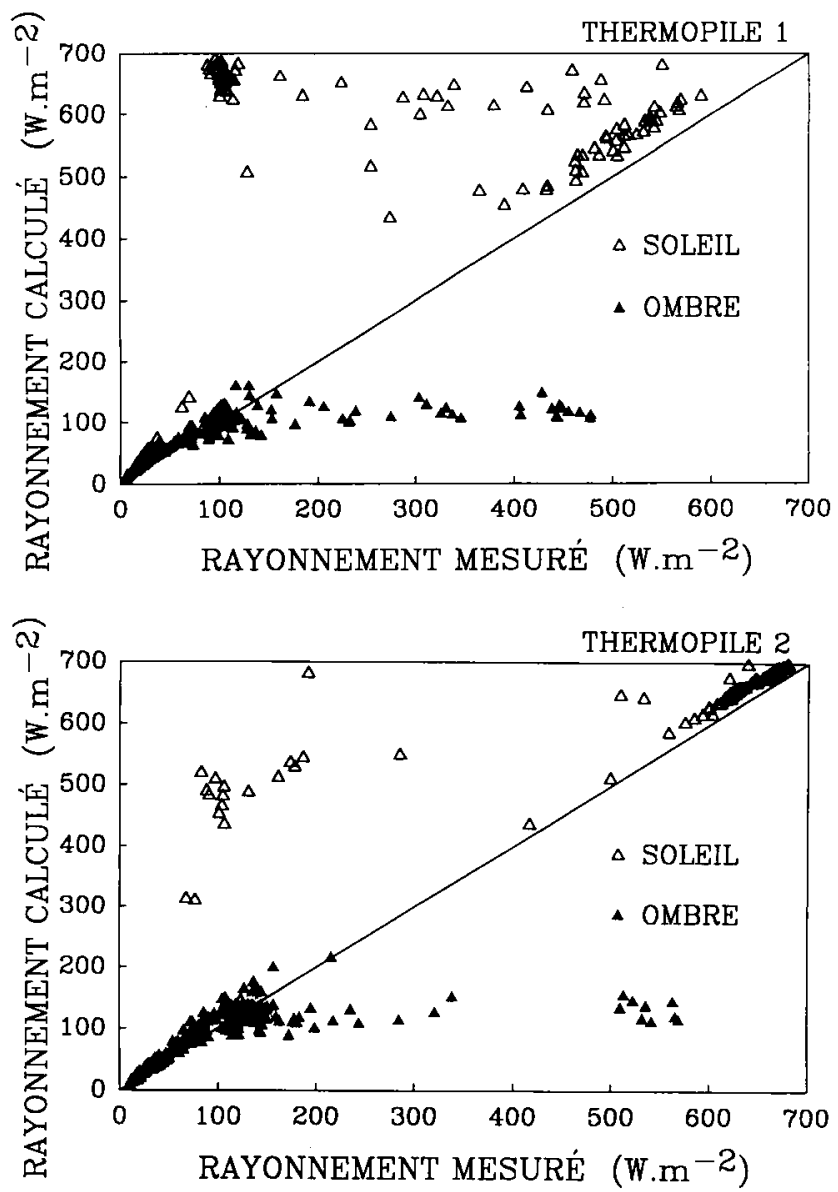

\section{Influence des pieds de vigne}

\section{Effet sur la transmission du rayonnement direct}

Dans le cas de cette vigne, le rapport du diamètre des ceps à la distance entre 2 pieds est égal à 0,06 , ce qui correspond à une porosité de 0,94 . La réduction du rayonnement direct par les seuls sarments peut dépasser $30 \%$ pour le capteur 1 qui est situé le plus près du rang (fig 9). L'influence sur les autres capteurs est moindre; la réduction ne dépasse $10 \%$ que lorsque les capteurs subissent l'ombrage de 2 rangs successifs, quand la hauteur du soleil est faible. À ce sujet, il faut noter que l'ombrage dû aux pieds de vigne varie fortement avec la course du soleil. La vigne étant orientée selon la direction nord-sud, l'ombrage augmente au cours de la matinée (azimuts du soleil négatifs) à cause du resserrement des ombres portées par les pieds. Puis subitement, l'ombrage disparaît car l'ombre portée n'atteint plus la bande de sol oc-
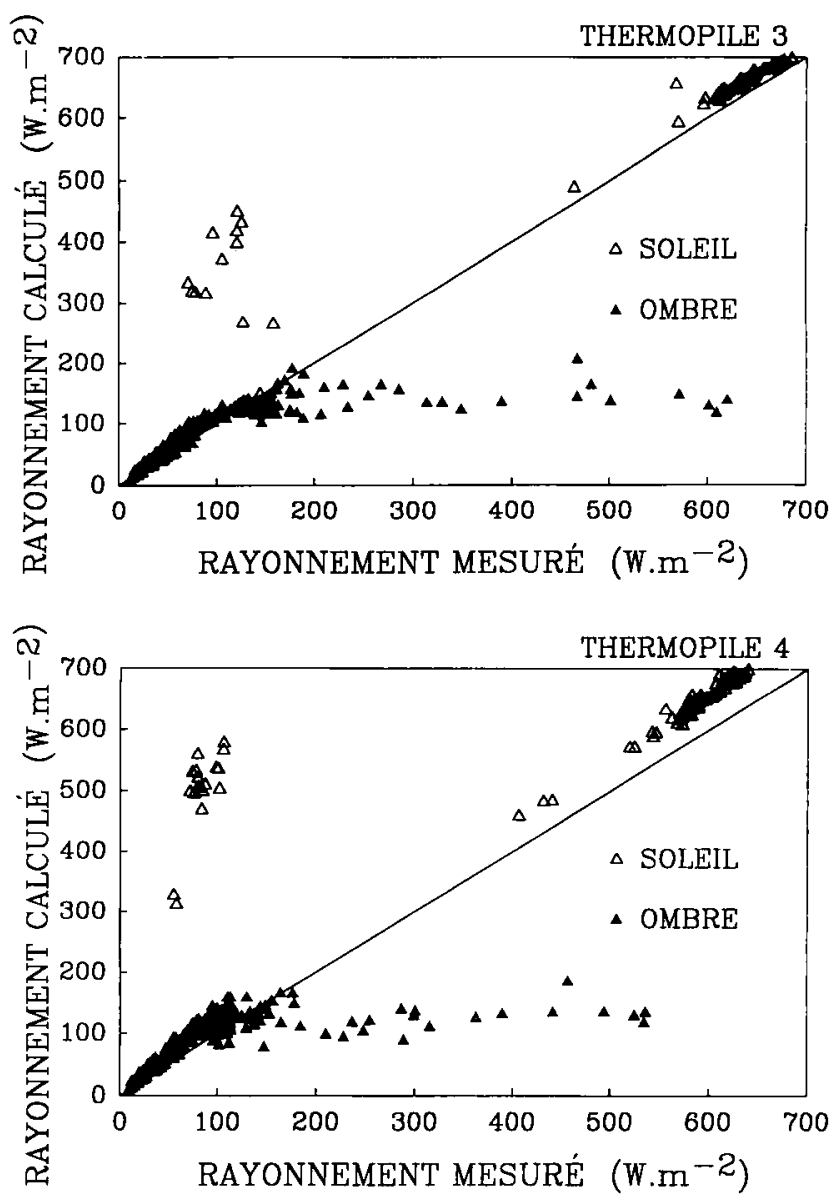

Fig 8. Comparaison des rayonnements transmis mesurés et simulés au niveau de chaque capteur : le capteur est considéré comme étant au soleil (resp à l'ombre) si le modèle indique que plus de $50 \%$ de la bande de sol qu'il occupe sont ensoleillés (resp ombragée). a) Thermopile 1, b) Thermopile 2, c) Thermopile 3, d) Thermopile 4. 
cupée par le capteur. De même, les brusques décrochements en début et en fin de journée correspondent à la disposition ou à l'apparition de l'ombrage d'une seconde rangée de pieds. Notons enfin qu'il existe des périodes assez longues où les bandes de sol ne subissent aucun ombrage des sarments.

La prise en compte de la réduction du rayonnement direct (ie, des surfaces ensoleillées) par les pieds se traduit par une légère amélioration de l'adéquation entre mesures et calculs (tableau II). Lorsque les parties ligneuses sont négligées, l'erreur de supposer que le capteur est au soleil alors qu'il est à l'ombre est plus fréquente que l'erreur inverse. De plus, cette dissymétrie est d'autant plus marquée que le capteur est placé près du rang de végétation. Lorsque la présence des sarments est prise en compte, le nombre

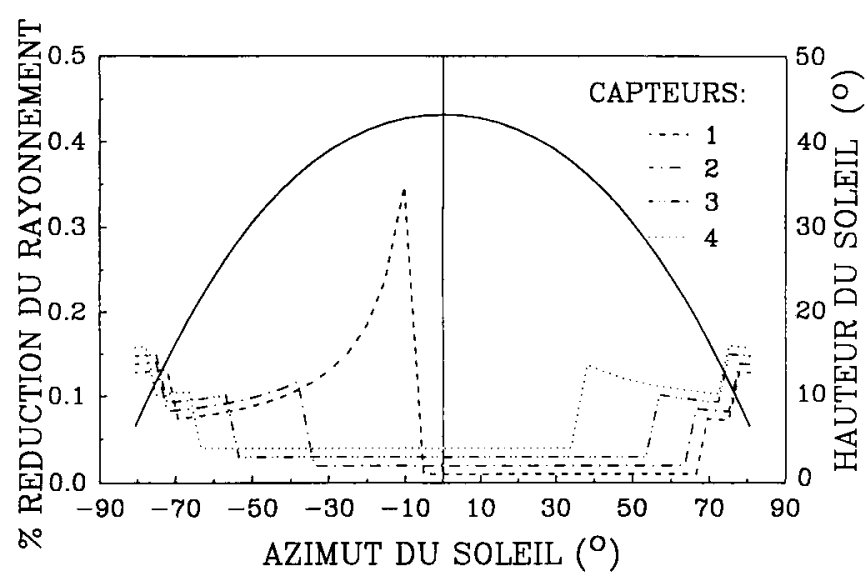

Fig 9. Évolution au cours de la journée de la réduction du rayonnement direct par l'ombrage des pieds de vigne sur les bandes de sol occupées par les capteurs. Les courbes sont décalées ( $n \%$ pour le capteur $n$ ) pour éviter leur superposition. L'évolution de la hauteur du soleil est indiqueee en trait plein.

Tableau II. Pourcentage du nombre de mesures répondant ou non à l'hypothèse sur le caractère totalement ensoleillé ou ombragé des capteurs de rayonnement transmis: OK: mesures répondant à I'hypothèse; Type $\mathrm{O} / \mathrm{S}$ : mesures où, à tort, le capteur est supposé être ombragé; Type S/O : mesures où, à tort, le capteur est supposé être au soleil.

\begin{tabular}{lcrrrrrr}
\hline \% de points & \multicolumn{2}{c}{ OK } & \multicolumn{3}{c}{ Type O/S } & \multicolumn{2}{c}{ Type S/O } \\
& $A$ & $B$ & $A$ & $B$ & $A$ & $B$ \\
\hline Thermopile 1 & 73,6 & 76,4 & 9,2 & 9,2 & 17,2 & 14,4 \\
Thermopile 2 & 88,0 & 88,8 & 4,8 & 4,8 & 7,2 & 6,4 \\
Thermopile 3 & 90,4 & 92,0 & 4,8 & 4,8 & 4,8 & 3,2 \\
Thermopile 4 & 88,0 & 88,8 & 5,2 & 5,2 & 6,8 & 6,0 \\
\hline
\end{tabular}

A : en négligeant l'effet des parties ligneuses; $B$ : en tenant compte de l'effet des parties ligneuses. de points de mesures satisfaisant l'hypothèse $\mathrm{H}_{2}$ est en légère hausse. Cette amélioration correspond exclusivement à une diminution des points supposés à tort être placés au soleil, et donc à une réduction de la dissymétrie entre les 2 types d'erreurs. Enfin, cet effet est surtout notable pour le capteur 1 , situé près de la ligne de pieds, alors qu'il est très faible pour les 3 autres.

\section{Effet sur la transmission du rayonnement diffus}

Les points de la figure 8 proches de la bissectrice montrent aussi une légère surestimation des rayonnements transmis calculés par le modèle. À priori, cette surestimation pourrait être attribuée en partie au support ligneux, puisqu'il réduit aussi le rayonnement diffus transmis au sol. En fait, l'influence des pieds de vigne sur la réduction du rayonnement diffus est assez faible: elle atteint une valeur maximale de $5 \%$ pour le capteur 1 et elle est de l'ordre de 1 à $2 \%$ pour les autres capteurs avec une valeur minimale pour le capteur 3 qui est le plus éloigné des rangs de végétation. Cette variation selon la distance au rang est tout à fait liée à celle de la surestimation du rayonnement transmis en fonction du capteur (ie, maximale pour le capteur 1 est minimale pour le capteur 3 , voir fig 8 ). De façon corollaire, l'influence des parties ligneuses sur la transmission du rayonnement diffus se traduit par une faible amélioration de l'adéquation mesures-calculs: pour le capteur le plus sensible (thermopile 1), les écarts sont réduits de $10 \mathrm{~W} \cdot \mathrm{m}^{-2}$ au maximum, et de $5 \mathrm{~W} \cdot \mathrm{m}^{-2}$ en moyenne.

D'autres faits peuvent également contribuer aux écarts entre mesures et modèle: en considérant un agencement du feuillage aléatoire et indépendant de la direction des rayonnements, le modèle peut ne pas très bien simuler l'extinction directionnelle du rayonnement dans la végétation. De plus, l'hypothèse d'agencement aléatoire a été vérifiée approximativement à partir de mesures de porosité en visée horizontale, alors que cette direction transporte peu d'énergie. Enfin, certains des paramètres du système ne sont pas très bien connus : la distribution spatiale et angulaire de la surface foliaire a été reconstituée à partir d'informations plus qualitatives que quantitatives; le rapport diffus/global a été supposé identique dans chacune des bandes spectrales alors que celui du domaine visible est 
généralement supérieur à celui du spectre solaire total (Bonhomme, 1970; Spitters et al, 1986).

\section{CONCLUSION}

L'application du modèle de transferts radiatifs au cas de la vigne traditionnelle s'est révélée satisfaisante à 2 points de vue. D'une part, les rayonnements simulés s'ajustent relativement bien aux mesures, même dans le cas de la distribution spatiale du rayonnement transmis au sol: la simulation de cette dernière pourrait d'ailleurs être utilisée pour déterminer à priori la disposition des capteurs au sol pour mesurer un rayonnement transmis moyen. D'autre part, certains points faibles du modèle et de la méthode de mesure ont été identifiés. Les plus importants sont les suivants :

- la comparaison entre calculs et mesures tend à prouver, une fois de plus, que l'agencement du feuillage varie avec la direction du rayonnement incident. Dès les premiers travaux, Nilson (1971) avait suggéré la possibilité d'un tel comportement, dont l'intégration dans la modélisation est d'ailleurs facile. Par contre, la difficulté intervient au niveau de la mesure des variations directionnelles du paramètre d'agencement, qui passe par la détermination expérimentale des fréquences de trous directionnelles. L'apparition de nouveaux appareils (Ceptomètre, Decagon; LAI2000, Li-cor) devrait cependant la faciliter;

- la mesure du rayonnement transmis au sol avec des capteurs ponctuels en position fixe est délicate: la surface réceptrice du capteur est le plus souvent soit complètement à l'ombre, soit totalement ensoleillée. Le capteur mesure donc rarement un rayonnement transmis moyen et l'hypothèse d'ergodicité n'est donc pas respectée. Cette difficulté est généralement résolue en utilisant des capteurs linéaires, ou des capteurs ponctuels mobiles, qui permettent une meilleure intégration spatiale du rayonnement transmis au sol (VarletGrancher et al, 1989);

- dans le cas de la vigne, les parties ligneuses (d'une façon générale, les organes autres que le feuillage) semblent avoir une influence sensible sur le bilan radiatif. Cet effet varie au cours du temps (interception du rayonnement direct en relation avec la course du soleil) et affecte la distribution spatiale du rayonnement transmis au sol. L'application du modèle à des couverts présentant des parties ligneuses importantes (vergers, forêts) devrait donc passer par la prise en compte de ces organes, comme le font déjà certains modèles de rayonnement appliqués aux couverts forestiers (Kurachi et al, 1986; Wang et Baldocchi, 1989).

En définitive, ce travail montre qu'il est permis d'élargir le champ d'application de ce modèle aux couverts présentant de fortes hétérogénéités spatiales.

\section{RÉFÉRENCES}

Acock B, Thornley JHM, Warren Wilson J (1969) Spatial variation of light in the canopy. In: Prediction and measurement of photosynthetic productivity, Proc IBP/PP Techn Meet, Trebon, 4-21 sept

Allen Jr LH (1974). Model of light penetration into a wide-row crop. Agron $J$ 66, 41-47

Anderson MC (1966) Stand structure and light penetration II. A theoretical analysis. J Appl Ecol 3, 41-54

Bonhomme R (1970) Composition spectrale des rayonnements solaire et céleste et énergie disponible pour les végétaux. Cah Assoc Fr Biométéorol 3, 23-31

Bonhomme R, Varlet-Grancher C (1977) Application aux couverts végétaux des lois de rayonnement en milieu diffusant. I. Établissement des lois et vérifications expérimentales. Ann Agron 28, 567-582

Chartier P (1966) Étude du microclimat lumineux dans la végétation. Ann Agron 17, 571-602

Cohen S, Fuchs M (1987) The distribution of leaf area, radiation: photosyntesis and transpiration in a Shamounti orange hedgerow orchard. Agric For Meteorol 40, 132-144

Fukai S, Loomis RS, (1976) Leaf display and light environments in row-planted cotton communities. Agric Meteorol 17, 353-379

Kurachi N, Hagihara A, Hozumi K (1986) Evaluation of the light interception by non-photosynthetic organs in a Larix leptolepis plantation. Ecol Res 1, 173-183

Monsi M, Saeki T (1953) Uber den Lichtfaktor in den Planzengesellscheften und seine Bedeutung fur die Stroffproduction. Jpn J Bot 14, 2252

Mutsaers HJW (1980) The effect of row orientation, date and latitude on light absorption by row crops. J Agric Sci Camb 95, 381-386 
Nilson T (1971) A theoretical analysis of the frequency of gaps in plant stands. Agric Meteorol $3,25-38$

Ozisik NM (1981) Radiative transfer. Wiley Interscience, New York

Prévot $L$ (1985) Modélisation des échanges radiatifs au sein des couverts végétaux, application à la télédétection, validation sur un couvert de maïs. Thèse d'Université, Paris VI, $178 \mathrm{p}$

Riou C, Valancogne C, Pieri P (1989) Un modèle simple d'interception du rayonnement solaire par la vigne - vérification expérimentale. Agronomie 9, 441-450

Sinoquet $H$ (1988) Modélisation des échanges radiatifs de courte longueur d'onde dans certains couverts hétérogènes: cultures en rangs, cultures associées en rangs alternés. Thèse de Docteur-Ingénieur, INA Paris-Grignon, $80 p+$ figures + annexes

Sinoquet H (1989) Modélisation de l'interception des rayonnements solaires dans une culture en rangs. I: Aspects théoriques. Agronomie 9, 125135

Sinoquet H, Bonhomme R (1989) Modélisation de l'interception des rayonnements solaires dans une culture en rangs. II: Structure géométrique du couvert et validation du modèle. Agronomie 9, 619-628

Smart RE (1973) Sunlight interception by vineyards. Am J Enol Vitic 24, 141-147

Spitters CJT, Toussaint HAJM, Goudriaan J (1986) Separating the diffuse and direct component of global radiation and its implications for modelling canopy photosynthesis. Part I. Components of incoming radiation. Agric For Meteorol 38, 217-229

Varlet Grancher C (1975) Variation et estimation de l'énergie d'origine solaire reçue sur des plans d'inclinaison et d'azimut variables. Ann Agron 26, 245-264

Varlet-Grancher C, Gosse G, Sinoquet H, Bonhomme R, Allirand JM (1989) Mise au point: rayonnement solaire absorbé ou intercepté par un couvert végétal. Agronomie 9, 3-21

Wang $H$, Baldocchi DD (1989) A numerical model for simulating the radiation regime within a deciduous forest canopy. Agric For Meteorol 46, 313-337

Warren Wilson J (1967) Stand structure and light penetration. III. Sunlit foliage area. J App/ Ecol $4,159-165$ 